\title{
An agent based model for assessing transmission dynamics and health systems burden for COVID-19
}

\author{
Narassima M. S. ${ }^{1}$, Anbuudayasankar S. P. ${ }^{2}$, Guru Rajesh Jammy ${ }^{3}$, Rashmi Pant ${ }^{4}$, \\ Lincoln Choudhury ${ }^{5}$, Aadharsh Ramakrishnan ${ }^{6}$, Denny John ${ }^{7}$ \\ ${ }^{1,2}$ Department of Mechanical Engineering, Amrita School of Engineering, Amrita Vishwa Vidyapeetham, \\ Coimbatore, India \\ ${ }^{3,4}$ Society for Health, Allied Research and Education (SHARE-INDIA), Telangana, India \\ ${ }^{5}$ Krashapana Consultancy Private limited, New Delhi, India \\ ${ }^{6}$ Department of Aerospace Engineering, Amrita School of Engineering, Amrita Vishwa Vidyapeetham, Coimbatore, India \\ ${ }^{7}$ Amrita Institute of Medical Sciences and Research Centre, Amrita Vishwa Vidyapeetham, Kochi, Kerala, India
}

\begin{tabular}{|c|c|}
\hline Article Info & ABSTRACT \\
\hline Article history: & Coronavirus disease of 2019 (COVID-19) pandemic has caused over \\
\hline Received Mar 11, 2021 & 230 million infections with more than 4 million deaths worldwide. \\
\hline Revised Oct 21,2021 & esearches have been using various mathematical and simulation techniques \\
\hline Accented Oct 27.2021 & to estimate the future trends of the pandemic to help the policymakers and \\
\hline Accepted Uct 27, 2021 & $\begin{array}{l}\text { healthcare fraternity. Agent-based models (ABM) could provide accurate } \\
\text { projections than the compartmental models that have been largely used. The }\end{array}$ \\
\hline Keywords: & from India to analyze the effects of interventions on the spread of the disease. \\
\hline Agent based model & A disease model with various states representing the possible progression of \\
\hline Coronavirus & $\begin{array}{l}\text { the disease was developed and simulated using AnyLogic. The results } \\
\text { indicated that imposing stricter non-pharmaceutical interventions (NPI) }\end{array}$ \\
\hline COVID-19 & lowered the peak values of infections, the proportion of critical patients, and \\
\hline SARS-CoV-2 & the deceased. Stricter interventions offer a larger time window for the \\
\hline Simulation & $\begin{array}{l}\text { healthcare fraternity to enhance preparedness. The findings of this research } \\
\text { could act as a start-point to understand the benefits of ABM-based models }\end{array}$ \\
\hline
\end{tabular}

This is an open access article under the $\underline{C C B Y-S A}$ license.

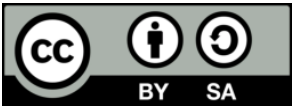

Corresponding Author:

Anbuudayasankar S. P.

Department of Mechanical Engineering, Amrita School of Engineering

Amrita Vishwa Vidyapeetham

Coimbatore, Tamil Nadu, India

Email: sp_anbu@cb.amrita.edu

\section{INTRODUCTION}

SARS-CoV-2 or Coronavirus disease of 2019 (COVID-19) layed its mark in India on January 30, 2020, during when World Health Organization (WHO) announced it was a pandemic [1]. It has created a global emergency by spanning across 221 territories and countries with 230,362,559 infections and 4,723,957 mortalities as of September 21, 2021 [2]. In India, the pandemic has caused 33,532,349 infections with 445,808 deaths and 32,776,358 recoveries as of September 21, 2021 [2]. This majorly bothers countries like India, which has a denser population. The transmission of the infection is governed by multiple parameters including the level of air pollution, viral load, presence of comorbidities, age, and others. Imposing restrictions to movement and imposing lockdowns to avoid crowding, unnecessary movements, and others could be some steadfast non-pharmaceutical interventions (NPIs) to curtail the spread of infection. 
Understanding the transmission mode, dynamics, and timeframe of infection are important to provide an effective response to the pandemic [3].

Simulation and modeling are proven techniques capable of producing accurate estimates by wiping out the investment of cost, time, and risks. In India, most of the research works have employed compartmental models to provide COVID-19 estimates. Modified versions of the susceptible (S), infected (I), and recovered $(\mathrm{R})$ models have been employed to add a few more states depending on study requirements. Susceptible (S), exposed (E), symptomatic (I), purely asymptomatic (P), hospitalized or quarantined (H), recovered $(\mathrm{R})$ and deceased (D) (SIPHERD) [4], susceptible (S), exposed (E), infective (I) and recovered (R) (SEIR) [5], [6], and a few analytical models [7], have been employed. As these models consider entities in each compartment to be homogeneous, they do not incorporate individual-level details. However, these can be achieved through the agent-based modeling (ABM) approach that provides the choice of defining agentlevel parameters and defining the interactions between them [8]. Owing to the evolving technological competencies and the ability of ABMs to deliver more accurate results, these have attracted researchers from several domains, in particular those like healthcare that demand a higher level of accuracy [9]. The bottom-up approach followed by these models works such that the behavior of the system is the resultant of those of the individual agents [10], [11]. Encompassment of such agent-level details and interactions make them more apt to be used for comprehending transmission of close contact infections.

ABMs have marked their presence to have been employed to address numerous problems relating COVID-19 such as effect of imposing and lifting of lockdowns and devising suitable intervention strategies [12]-[16], maintaining self-hygiene [14], [16], [17], transmission dynamics from infected people (man-man) and objects (airborne particulates) [14], time-dependent contacts [14], [16], shielding the vulnerable cohorts [16], [18], contact tracking at different locations [13], [17], [18], effect of viral load [17], genomic sequencing [19], and others. ABM-based recommendations for capacity planning of healthcare systems in India to handle the surge of infections and hospitalization was presented together by the center for disease dynamics, economics and policy (CDDEP) and Princeton University [20]. Models that represent the entire nation would be less helpful for policy-making as most of the strategies are devised locally based on the severity of infection. The methodologies of these studies with those that focus on individuals such as assessing the user experience [21], interactive chatbots to tackle mental health [22], wearable devices for real-time monitoring [23], and others, could help in lower-level analysis. It is more practical to tailor-make the interventions as some particularities such as migrant population, contact pattern, type of employments/ occupation, and others vary with location. It is evident from the literature that though ABMs can include granular information, technological capabilities need to be well developed. This study involves an ABM approach to compare the effects of various NPI scenarios on the spread of infection using a synthetic population from Telangana state, India. The study could provide a framework to help the decision-making using localized parameters.

\section{RESEARCH METHOD}

\subsection{Study design}

The area under study was a part of the Rangareddy district comprising of 5,48,323 agents. AnyLogic 8.5.2 University edition was used to develop and simulate the ABM [24]. The study adheres to the standards of the international society for pharmacoeconomics and outcomes research (ISPOR-SMDM) modeling good research practices and ethical good practice in modeling [25], [26]. In this way, the variables chosen for study, scope, assumptions, and shortcomings were made clear to the readers.

\subsection{Synthetic population}

Creating a synthetic population is classified among the successful techniques to represent a population whilst maintaining the confidentiality of each person. Synthetic populations are statistically comparable with the corresponding actual populations that they represent [27]. This study uses 5,48,323 agents that represent $10.35 \%$ of Rangareddy's population $(52,96,741)$ according to Census of India 2011 . To present age-wise discrimination, the population was split into three groups consisting of people aged less than 5, from 5 to 59, and 60 and above consisting of 47,039, 4,59,372, and 41,912 agents respectively.

\subsection{Transmission rates of COVID-19}

The transmission rate used in the study ranged from 1 to 10 percent as reported by the WHO (16-24 Feb 2020) [28]. Lockdowns reduce the number of contacts made with agents but have no effect on the transmission rate. But, NPIs such as mask or social distancing could reduce the transmission rate directly. 


\subsection{Contact network}

The transmission dynamics is governed by the contact network. The study conducted by Kumar et al. in Ballabgarh, India aimed to define the contact rate that would be useful for the study of closecontact infections [29]. This was used as a reference to define contact rates for the present study. Arena's (Version 16.00.00002) 'Input Analyzer' was used to derive the distributions for the number of people contacted by people of each age-groups. However, there are errors linked to these distributions generated as shown in Table 1.

Table 1. Contact rate distribution of Ballabgarh [29]

\begin{tabular}{ccccccc}
\hline Age & $\begin{array}{c}\text { Population } \\
\text { size }\end{array}$ & $\begin{array}{c}\text { Average contacts } \\
\text { made each day }\end{array}$ & Minimum & Maximum & Contact rates' distribution & $\begin{array}{c}\text { Square Error } \\
\left(\mathrm{x} 10^{-3}\right)\end{array}$ \\
\hline Less than 5 & 378 & 15.11 & 4 & 48 & Norm $(15.1,6.82)$ & 6.6 \\
5 to 59 & 2185 & 17.19 & 1 & 67 & Norm $(17.2,8.01)$ & 3.2 \\
60 and above & 380 & 12.86 & 1 & 41 & Norm $(12.9,5.49)$ & 2.3 \\
\hline
\end{tabular}

The proportion of population across the townships in India was used to derive the population densities as shown in Table 2 [30]. These were used to define the contact rates for these townships assuming a density-dependent (DD) contact rate using Ballabgarh's population density (551 people/ $\mathrm{km}^{2}$ ) as reference [29]. The slope in (1) is used to derive the contact rates for each age group based on the product of the proportion of the population in each township weighed according to their corresponding multiplication factors (Table 2). These multiplication factors were multiplied by the number of people contacted by each person and the resultant dataset was passed into the input analyzer to get the distribution followed. Lognormal was found to be the best fit distribution as shown in Table 3.

$$
\text { Contact rate }=\text { Slope } \times \frac{\text { Population }}{\text { Area }}
$$

Table 2. Indian township classification with population statistics [30]

\begin{tabular}{lccc}
\hline Census classification & Population density per km2 & Proportion of Indian population (\%) & Multiplication factor \\
\hline Statutory town & 3977 & 26.3 & 7.22 \\
Census town & 2069 & 4.5 & 3.75 \\
Outgrowth & 1241 & 0.4 & 2.25 \\
Village & 292 & 68.9 & 0.53 \\
\hline
\end{tabular}

Table 3. Parameters for the model

\begin{tabular}{|c|c|c|}
\hline Variables & $\begin{array}{ll}<5 & 5-59 \\
\end{array}$ & References \\
\hline \multirow{3}{*}{ Contacts made each day (age-wise) } & Lognormal $(2.77 .0 .90,6)$ & [29], [30] \\
\hline & $\operatorname{Lognormal}(2.88,0.86,6)$ & \\
\hline & $\operatorname{Lognormal}(2.60,0.84,5)$ & \\
\hline Transmission rate upon contact & Random (1 to 10 ) percent & [28] \\
\hline Asymptomatic infections (\%) & 80 & [31] \\
\hline Incubation period (days) & 5 & {$[11],[3]$} \\
\hline Duration of treatment (days) & 14 & [3] \\
\hline Critical cases requiring intensive care unit (ICU) $(\%)$ & 11 & {$[32],[33]$} \\
\hline Length of treatment in ICU (days) & Triangular $(7,8,9)$ & {$[3],[33]$} \\
\hline Patients who require ventilator support (\%) & 88 & [33] \\
\hline Duration of ventilator support (days) & Triangular $(5,7,12)$ & [3] \\
\hline Duration from arrival of symptom to admission (no intervention; days) & 5 & [34] \\
\hline Duration from arrival of symptom to admission (with intervention; days) & 3 & [34] \\
\hline Proportion of deceased people & Number of deaths/ Number of infections & {$[2]$} \\
\hline
\end{tabular}

\subsection{State chart}

The different states of existence of an agent are presented in the state chart as shown in Figure 1 that was developed using AnyLogic [35]. Initially, all agents are assumed to be healthy. Introduction of an infection results in transmission of infection to other agents upon contact. Infected agents become either asymptomatic or symptomatic. The former is untraceable and continue to infect healthy agents till they recover whilst the latter get isolated upon admission post their incubation period. Admitted agents recover or decease while present in admitted, ICU, or ventilator states [10]. 


\subsection{Model parameters}

The variables used for the simulation have been gathered from various published sources such as manuscripts, reports, and pre-prints. As the research was conducted during the earlier phases, not all the variables could be collected from the region under study. However, some variables such as contacts per day, incubation period, asymptomatic infections, and others remain to be almost the same across multiple geographies.

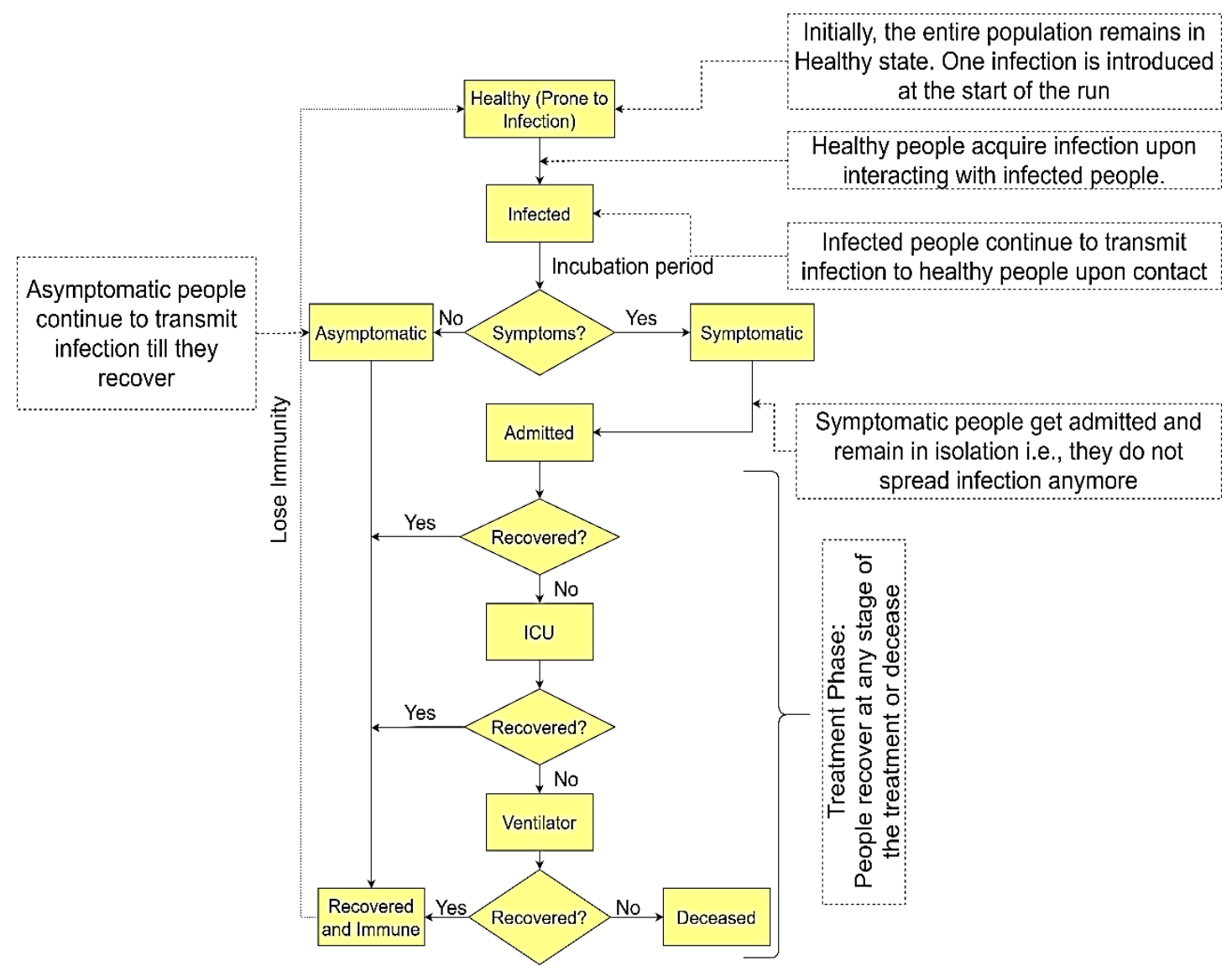

Figure 1. State chart for agent(s) (people)

\subsection{Model scenarios}

Three scenarios were simulated over 365 days to analyze the effect of lockdowns on the spread of infection within the population. The rate of spread is proportional to the number of interactions within the population forms the basic idea of these scenarios. There is no NPI introduced in the first scenario that depicts a "no lockdown" condition. The next two scenarios that involve lockdowns necessitate a reduction in the number of contacts. The findings of a study by Prem et al. viz. number of interactions by individuals of each age-groups across various places such as school, office, home, and others, were used to develop the same [36]. The contacts made at school were eliminated to enact the NPI scenarios whilst reducing the contacts at the office and other locations by $50 \%$ and $75 \%$ for the second and third scenarios respectively. The results of these scenarios have been compared to comprehend the effects of interventions.

\section{RESULTS AND DISCUSSION}

The models were run separately for all three age groups for all the scenarios. The results indicated a similar trend for all the cohorts with differences in magnitude and longevity. An abrupt decline in the rate of spread, the number of admissions, and critical infections can be seen as the stringency of lockdown is increased (Figure 2 and Figure 3). Detailed output (age-wise), AnyLogic model file, synthetic population, and input spreadsheet can be accessed from the repository [37]. Also, the time taken to attain the peak is prolonged by imposing stricter lockdowns. 
The number of people uninfected is higher with the increase in stringency of lockdowns as seen in Figure 2(a). Upon simulation for 365 days, the proportions of uninfected people as compared to the initial population are 28.53, 76.33, and 93.8 percent respectively for the no lockdown, 50 percent, and 75 percent scenarios. The peak infections attained in scenarios 2 and 3 (Figure 2(b)) are 1,29,779 and 33,973 those which correspond to the infections that would have occurred in 33 and 25 days in the first scenario. The peak infections in the three scenarios are 191,907, 37,790, and 7,986 that represent 35, 6.89, and 1.46 percent of the initial population respectively, as shown in Figure 2(c). The corresponding deaths in Figure 2(d) indicate $0.42,0.14$, and 0.04 percent respectively of the initial population for the three scenarios.

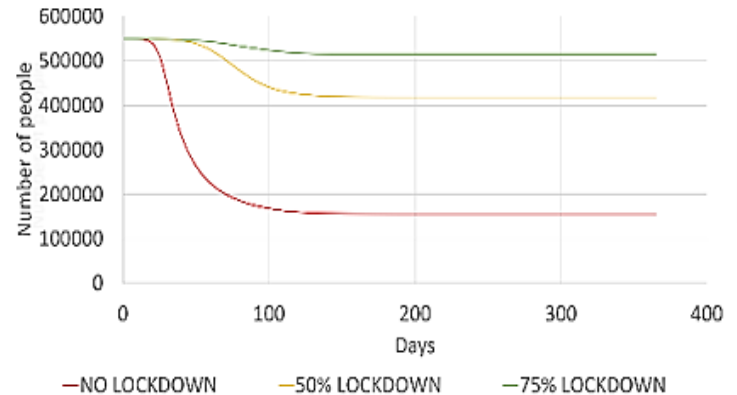

(a)

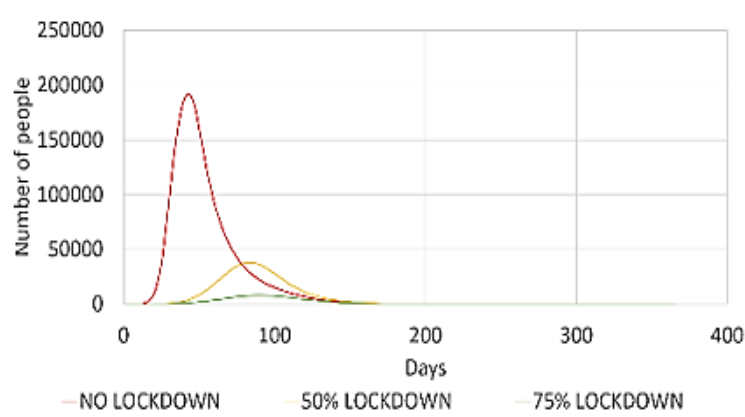

(c)

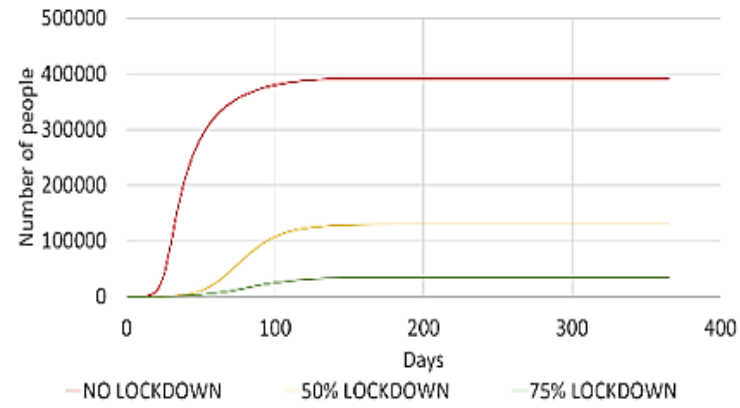

(b)

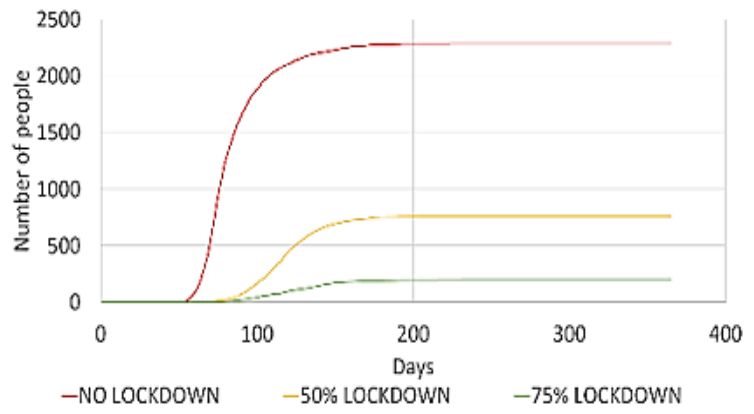

(d)

Figure 2. (a) Number of uninfected people, (b) Cumulative infections, (c) Number of infected people (for a given instant), and (d) Number of people deceased

The peaks of asymptomatic people for the three scenarios in Figure 3(a) correspond to 31.71, 6.28, and 1.33 percent of the initial population respectively. The peak values of the three scenarios are 17,3892, 34,414 , and 7,281 that are reached on $44^{\text {th }}, 84^{\text {th }}$, and $90^{\text {th }}$ days respectively for the three scenarios. A similar trend is observed in the peak values of admitted people in Figure 3(b) that represent 6.42, 1.33, and 0.28 of the initial population for the three scenarios. Similar trends are observed across the three scenarios with respect to the peak numbers in ICU (Figure 3(c)) and ventilator (Figure 3(d)) supports. The 2,390, 496, and 94 are the peak admissions in ICU, and 1,929, 405, and 78 are the peak number of patients requiring ventilator support respectively for the three scenarios.

Peak values attained at each state are presented in Table 4. Percentage (\%) values in the table correspond to the proportion of the initial healthy population. Comparing the values of the first scenario with the corresponding values of the other two scenarios indicate that there is a proportionate decrease in the peak values with an increase in the stringency of lockdown. Comparing the results of the first and third scenarios reveal an apparent decrease in the peaks of ICU admissions and people requiring ventilator support from 2,390 to 94 and from 1,929 to 78 respectively. 


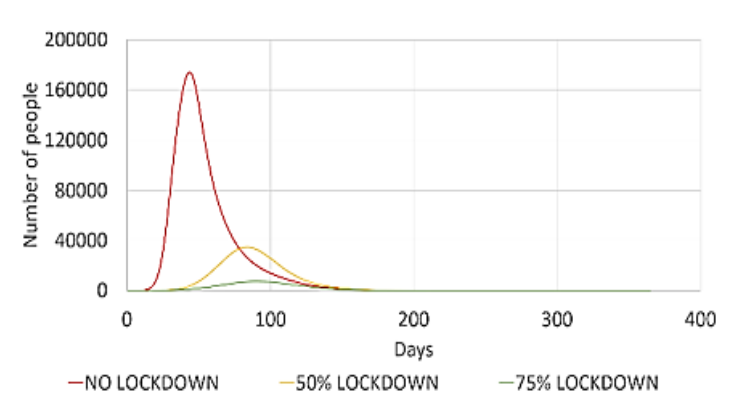

(a)

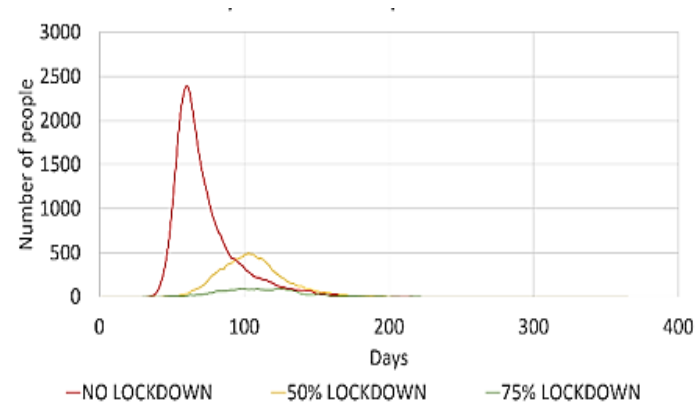

(c)

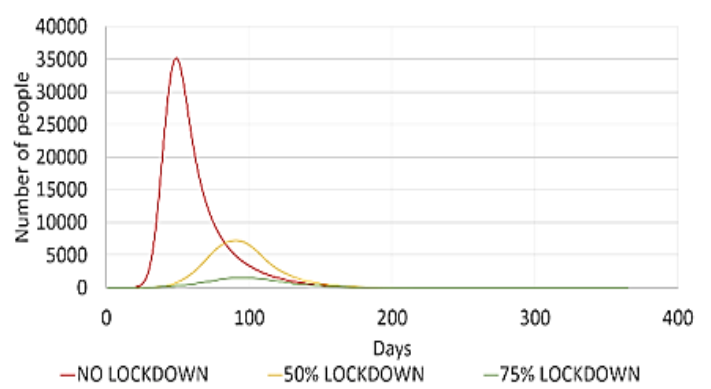

(b)

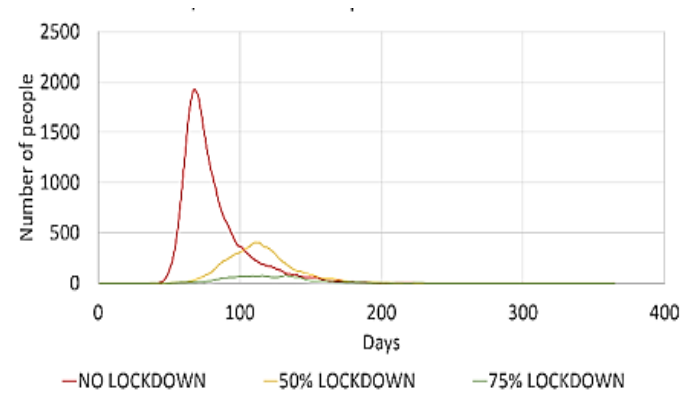

(d)

Figure 3. (a) Number of asymptomatic people, (b) Number of admitted people, (c) Number of people in ICU, and (d) Number of people on ventilators

Table 4. Peak values for various health conditions

\begin{tabular}{|c|c|c|c|c|c|c|c|c|c|}
\hline & \multicolumn{3}{|c|}{ No lockdown } & \multicolumn{3}{|c|}{$50 \%$ lockdown } & \multicolumn{3}{|c|}{$75 \%$ lockdown } \\
\hline & Value & $\%$ & Day & Value & $\%$ & Day & Value & $\%$ & Day \\
\hline Uninfected & 548,320 & 100 & 1 & 548,321 & 100 & 1 & 548,321 & 100 & 1 \\
\hline Infected & 191,907 & 35.00 & 43 & 37,790 & 6.89 & 84 & 7,986 & 1.46 & 90 \\
\hline Asymptomatic & 173,892 & 31.71 & 44 & 34,414 & 6.28 & 84 & 7,281 & 1.33 & 90 \\
\hline Symptomatic & 23,269 & 4.24 & 36 & 3,450 & 0.63 & 78 & 723 & 0.13 & 85 \\
\hline Admitted & 35,186 & 6.42 & 49 & 7,271 & 1.33 & 91 & 1,510 & 0.28 & 97 \\
\hline ICU & 2,390 & 0.44 & 60 & 496 & 0.09 & 104 & 94 & 0.02 & 125 \\
\hline Ventilator & 1,929 & 0.35 & 68 & 405 & 0.07 & 114 & 78 & 0.01 & 132 \\
\hline Deceased & 2,288 & 0.42 & 224 & 760 & 0.14 & 209 & 199 & 0.04 & 231 \\
\hline Recovered & 389,572 & 71.05 & 226 & 129,018 & 23.5 & 216 & 33,773 & 6.16 & 230 \\
\hline
\end{tabular}

\section{SENSITIVITY ANALYSIS}

Healthcare systems, governments, and the general public have all been affected in one way or another by the pandemic. Incorporating multiple shielding mechanisms as described by the Swiss Cheese model has proven effective in controlling the spread of infection [38]. There have been several interventions in unison to combat the infection such as the use of face masks, sanitizers, following social distancing, avoiding mass gatherings, lockdowns, and others [38]. The incubation period of infected patients in India is seen to be longer (6.93 days) according to recent researches [39] with a larger proportion of the infected being asymptomatic (91\%) [40]. The incubation period and proportion of asymptomatic infections have been altered to perform sensitivity analysis (SA) across five scenarios as shown in Figure 4(a)-(e). The number of people infected (Figure 4(a)), admitted (Figure 4(b)), in ICU (Figure 4(c)), and with ventilator support (Figure 4(d)) have increased substantially with increase in incubation days and asymptomatic proportion. 'A' and 'IN' in the notation of scenario names correspond to the incubation days and proportion of asymptomatic infections respectively (Figure 4(e)).

SA shows that there is a higher number of infections in scenarios with longer incubation days. This increases the duration between exposure to admission (symptomatic) or recovery (asymptomatic) during when people transmit the infection. Higher asymptomatic infections are of serious concern as it increases untraceable transmissions. This demands the requirement of more contact tracking measures to isolate the potential spreaders, the asymptomatic people. A higher proportion of asymptomatic infections are the reason for the drop in admissions in the scenarios IN7A91 and IN5A91. These conservative estimates aim to improve the capacity planning of healthcare systems to manage similar events in the future. The variations 
that could possibly happen in the rate of transmission and severity of the infection because of variations in mutations and external factors are to be explored to anticipate the required changes [41].

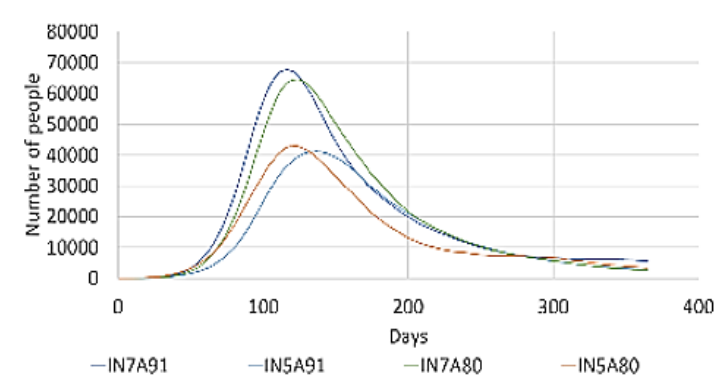

(a)

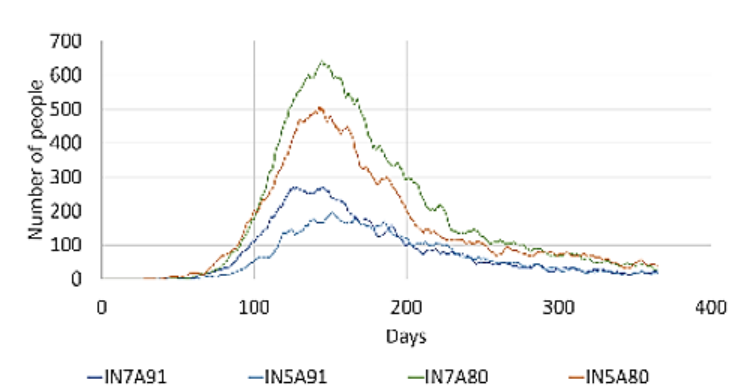

(c)

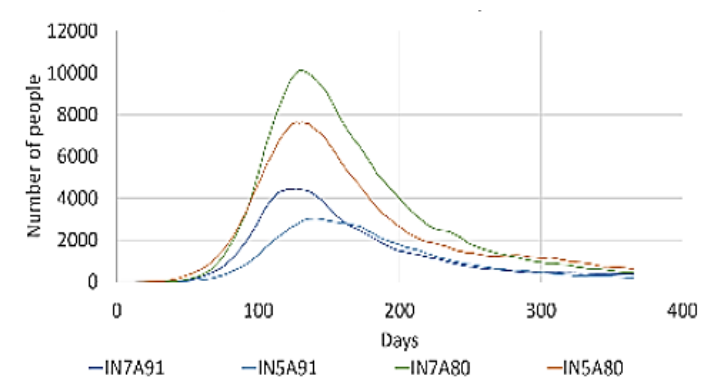

(b)

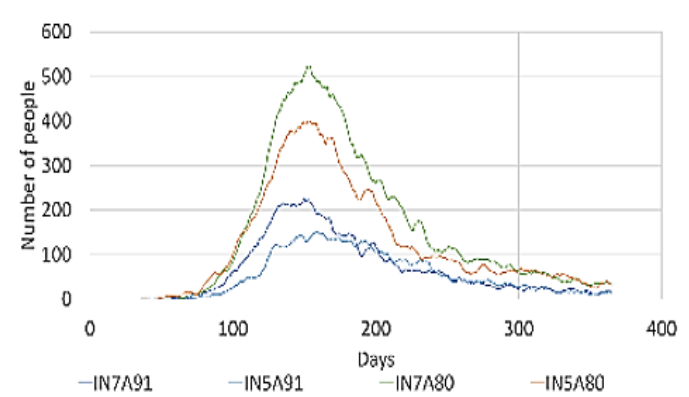

(d)

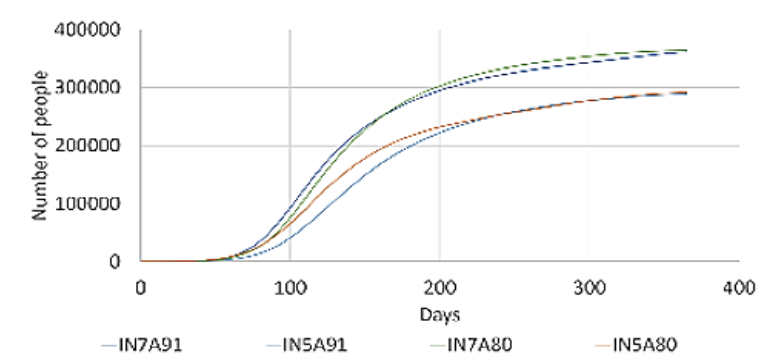

(e)

Figure 4. Sensitivity analysis across five scenarios; (a) number of infected people, (b) number of admitted people, (c) number of people in icu, (d) number of people on ventilator, and (e) number of cumulative infections

\section{CONCLUSION}

ABM-based models have aimed to address smaller geographical territories as their core strength lies in focusing at the agent level. Agents with similar characteristics and parameters such as age, gender, health conditions, work culture, geographical location, and others, are more likely to be handled in a similar pattern with variations depending on the differences in their characteristics. The present study used a synthetic population from Rangareddy, Telangana state, India to simulate the developed ABM to analyze the effects of various NPIs on the spread of infection. Such local studies are of practical interest for the decision-makers to implement localized interventions as not all interventions are suitable on a large scale. The main focus was to provide a framework that explains how ABMs could be useful in projecting the spread of infectious diseases across geography. This can be extended to estimate the effects of one or more interventions on the spread to assist policymakers and preparedness of healthcare systems. A total of 5,43,823 agents split into three different age groups were simulated for the three scenarios that depict a no lockdown, 50 percent, and 75 percent lockdown conditions. Several variables were used to simulate the model. Results revealed significant reductions in infections and mortalities upon imposing stricter lockdowns which was evident through a clear decline in the number of infections, hospitalizations, and critical infections. The reduction in peak values and delay in the occurrence of the peak values also indicated the effectiveness of lockdowns. SA revealed that a higher number of asymptomatic infections could lead to a proportionately higher rate of spread. ISPORSMDM Modeling Good Research Practices and ethical good practices in modeling have been adhered to throughout the study. 
ABMs have helped analyze several scenarios concerning COVID-19 including the comparison of a no lockdown and varied lockdown scenarios similar to that in this study. Apart from this, some of the explored dimensions include decreasing the contacts made outside the close circle, age-dependent lockdowns, tracking of symptomatic people, isolating the vulnerable population, following self-hygiene practices such as social distancing, masks, and others. In order to incorporate the effect of lockdowns, the number of contacts made outside the home was reduced proportionately. The increase in stringency of lockdowns not only reduced the peaks but also delayed their occurrences thereby providing more time window to plan their capacities to accommodate the surge of infections. This signifies the necessity to minimize the interactions among the people to flatten the curve.

However, variables such as following social distancing, using face masks, gradual lifting of lockdowns, presence of comorbidities, and others have not been included in this model. Contact networks and population dynamics of specific territories need to be explored fully to produce accurate estimates. The effect of lockdowns on mental health could be explored to identify the causes and effects and the most vulnerable cohorts. These could help practitioners to connect with the target groups to keep them motivated to improve their psychological health. Use of wearable gadgets paired with mobile phones or those that facilitate real-time monitoring might be handy for the caretakers and practitioners. The variables used for the simulation do not correspond to those of Rangareddy which might causes deviations in some estimates. In addition, traditional ayurvedic medicine that is used as antivirals could help to combat the surge of infections effectively.

\section{REFERENCES}

[1] WHO, WHO announces COVID-19 outbreak a pandemic, 2020. Accessed: Nov. 09, 2021. [Online]. Available: https://www.euro.who.int/en/health-topics/health-emergencies/coronavirus-covid-19/news/news/2020/3/whoannounces-covid-19-outbreak-a-pandemic

[2] Worldometer - Coronavirus Update (Live), Worldometer, 2020. Accessed: Apr. 12, 2020. [Online]. Available: https://www.worldometers.info/coronavirus/

[3] W. Zhao, X. Zha, N. Wang, D. Li, A. Li, and S. Yu, "Clinical characteristics and durations of hospitalized patients with COVID-19 in Beijing: a retrospective cohort study," medRxiv, 2020, doi: 10.1101/2020.03.13.20035436.

[4] A. Mahajan, N. A. Sivadas, and R. Solanki, "An epidemic model SIPHERD and its application for prediction of the spread of COVID-19 infection in India," Chaos, Solitons and Fractals, vol. 140, 2020, doi: 10.1016/j.chaos.2020.110156.

[5] V. R. Verma, A. Saini, S. Gandhi, U. Dash, and S. F. Koya, "Capacity-need gap in hospital resources for varying mitigation and containment strategies in India in the face of COVID-19 pandemic," Infect. Dis. Model., vol. 5, no. 1, pp. 608-621, 2020, doi: 10.1016/j.idm.2020.08.011.

[6] Z. Zhang and S. Jain, "Mathematical model of Ebola and Covid-19 with fractional differential operators: NonMarkovian process and class for virus pathogen in the environment," Chaos, Solitons and Fractals, vol. 140, no. 1, 2020, doi: 10.1016/j.chaos.2020.110175.

[7] B. Ambikapathy and K. Krishnamurthy, "Mathematical modelling to assess the impact of lockdown on COVID-19 transmission in India: Model development and validation," JMIR Public Health Surveill., vol. 6, no. 1, 2020, doi: $10.2196 / 19368$

[8] S. Gallagher Advisor and W. F. Eddy, "Comparing compartment and agent-based models," Joint Statistical Meeting, Baltimore, 2017.

[9] V. Grimm, U. Berger, D. L. DeAngelis, J. Gary Polhill, J. Giske, and S. F. Railsback, "The ODD protocol: A review and first update," Ecological Modelling, vol. 221, no. 23, pp. 2760-2768, 2010, doi: 10.1016/j.ecolmodel.2010.08.019.

[10] J. Gomez, J. Prieto, E. Leon, and A. Rodriguez, "INFEKTA: A General Agent-based Model for Transmission of Infectious Diseases: Studying the COVID-19 Propagation in Bogotá - Colombia," medRxiv, 2020, doi: 10.1101/2020.04.06.20056119.

[11] S. L. Chang, N. Harding, C. Zachreson, O. M. Cliff, and M. Prokopenko, "Modelling transmission and control of the COVID-19 pandemic in Australia," Nature Communications, vol. 11, no. 1, pp. 1-13, 2020, doi: 10.1038/s41467-020-19393-6.

[12] M. Tracy, M. Cerdá, and K. M. Keyes, "Agent-Based Modeling in Public Health: Current Applications and Future Directions," Annual review of public health, vol. 39, pp. 77-94, 2018, doi: 10.1146/annurev-publhealth-040617014317.

[13] M. S. Shamil, F. Farheen, N. Ibtehaz, I. M. Khan, and M. S. Rahman, "An Agent Based Modeling of COVID-19: Validation, Analysis, and Recommendations," medRxiv, 2020, doi: 10.1101/2020.07.05.20146977.

[14] M. Jalayer, C. Orsenigo, and C. Vercellis, "CoV-ABM: A stochastic discrete-event agent-based framework to simulate spatiotemporal dynamics of COVID-19," arXiv preprint arXiv:2007.13231, Jul. 2020.

[15] N. M. Gharakhanlou and N. Hooshangi, "Spatio-temporal simulation of the novel coronavirus (COVID-19) outbreak using the agent-based modeling approach (case study: Urmia, Iran)," Informatics Med. Unlocked, vol. 20, 2020, doi: 10.1016/j.imu.2020.100403. 
[16] N. Hoertel et al., "A stochastic agent-based model of the SARS-CoV-2 epidemic in France," Nat. Med., vol. 26, pp. 1417-1421, 2020, doi: 10.1038/s41591-020-1001-6.

[17] C. C. Kerr et al., "Covasim: an agent-based model of COVID-19 dynamics and interventions," medRxiv, 2020, doi: 10.1101/2020.05.10.20097469.

[18] N. Hoertel et al., "Facing the COVID-19 epidemic in NYC: a stochastic agent-based model of various intervention strategies," medRxiv, 2020, doi: 10.1101/2020.04.23.20076885.

[19] R. J. Rockett et al., "Revealing COVID-19 transmission in Australia by SARS-CoV-2 genome sequencing and agent-based modeling," Nature Medicine, vol. 26, pp. 1398-1404, 2020, doi: 10.1038/s41591-020-1000-7.

[20] K. Tseng, I. Frost, G. Kapoor, A. Sriram, A. Nandi, and R. Laxminarayan, Covid-19 India: State-level Estimates of Hospitalization Needs, CDDEP and Princeton University, 2020.

[21] A. M. Saleh, H. Y. Abuaddous, O. Enaizan, and F. Ghabban, "User experience assessment of a COVID-19 tracking mobile application ( AMAN ) in Jordan," Indonesian Journal of Electrical Engineering and Computer Science, vol. 23, no. 2, pp. 1120-1127, 2021, doi: 10.11591/ijeecs.v23.i2.pp1120-1127.

[22] I. Salhi, K. El Guemmat, M. Qbadou, and K. Mansouri, "Towards developing a pocket therapist : an intelligent adaptive psychological support chatbot against mental health disorders in a pandemic situation," Indonesian Journal of Electrical Engineering and Computer Science, vol. 23, no. 2, pp. 1200-1211, 2021, doi: 10.11591/ijeecs.v23.i2.pp1200-1211.

[23] M. S. Narassima, S. P. Anbuudayasankar, S. K. Vasudevan, and J. Abhinavaram, "Physicians' and users' perceptions towards wearable health devices," Indonesian Journal of Electrical Engineering and Computer Science, vol. 5, no. 1, pp. 234-242, 2017, doi: 10.11591/ijeecs.v5.i1.pp234-242.

[24] R-H. Bai, W-Y. Dong, Y. Shi, A-Z. Feng, A-D. Xu, and J. Lyu, "Simulation of epidemic trends for a new coronavirus under effective control measures," New Med., vol. 30, no. 2, pp. 8-12, 2020, doi: 10.12173/j.issn.10045511.2020.02.03.

[25] J. J. Caro, A. H. Briggs, U. Siebert, and K. M. Kuntz, "Modeling Good Research Practices-Overview: A Report of the ISPOR-SMDM Modeling Good Research Practices Task Force-1," vol. 32, no. 5, pp. 667-677, 2012, doi: $10.1177 / 0272989 X 12454577$.

[26] L. A. Boden and I. J. McKendrick, "Model-based policymaking: A framework to promote ethical 'good practice' in mathematical modeling for public health policymaking," Front. Public Heal., vol. 5, pp. 1-7, 2017, doi: 10.3389/FPUBH.2017.00068.

[27] A. Adiga et al., "Generating a synthetic population of the United States *," 2015.

[28] Report of the WHO-China Joint Mission on Coronavirus Disease 2019 (COVID-19), 2020. [Online]. Available: https://www.who.int/docs/default-source/coronaviruse/who-china-joint-mission-on-covid-19-final-report.pdf

[29] S. Kumar et al., "Who interacts with whom? Social mixing insights from a rural population in India," PLoS One, vol. 13, no. 12, Dec. 2018, doi: 10.1371/journal.pone.0209039.

[30] D. Balk, M. R. Montgomery, H. Engin, N. Lin, E. Major, and B. Jones, "Urbanization in India: Population and urban classification grids for 2011," Data, vol. 4, no. 1, pp. 1-16, 2019, doi: 10.3390/data4010035.

[31] M. Day, "Covid-19: four fifths of cases are asymptomatic, China figures indicate," BMJ, vol. 369, 2020, doi: 10.1136/bmj.m1375.

[32] M. Arentz et al., "Characteristics and Outcomes of 21 Critically Ill Patients with COVID-19 in Washington State," JAMA, vol. 323, no. 16, pp. 1612-1614, 2020, doi: 10.1001/jama.2020.4326.

[33] G. Grasselli et al., "Baseline Characteristics and Outcomes of 1591 Patients Infected with SARS-CoV-2 Admitted to ICUs of the Lombardy Region, Italy," JAMA, vol. 323, no. 16, pp. 1574-1581, 2020, doi: 10.1001/jama.2020.5394.

[34] World Health Organization, WHO Coronavirus disease 2019 (COVID-19) Situation Report - 73, 2020. [Online]. Available: https://apps.who.int/iris/handle/10665/331686

[35] I. Grigoryev, Anylogic in three days: A quick course in simulation modeling, Oakbrook Terrace, USA: TheAnyLogic Company, 2018.

[36] K. Prem, A. R. Cook, and M. Jit, "Projecting social contact matrices in 152 countries using contact surveys and demographic data," PLoS Comput. Biol., vol. 13, no. 9, 2017, doi: 10.1371/journal.pcbi.1005697.

[37] M. S. Narassima et al., "An Agent Based Model for assessing spread and health systems burden for Covid-19 using a synthetic population in Rangareddy district, Telangana state, India," figshare, 2021, doi: 10.6084/m9.figshare.16960201.v1

[38] J. Y. Noh, J. Y. Song, J. G. Yoon, H. Seong, H. J. Cheong, and W. J. Kim, "Safe hospital preparedness in the era of COVID-19: The Swiss cheese model," International Journal of Infectious Diseases, vol. 98, pp. 294-296, Sep. 2020, doi: 10.1016/j.ijid.2020.06.094.

[39] S. Patrikar et al., "Incubation Period and Reproduction Number for novel coronavirus (COVID-19) infections in India," medRxiv, Jun. 2020, doi: 10.1101/2020.06.27.20141424.

[40] N. Kumar et al., "Epidemiology of SARS-CoV-2 infection in Karnataka State, South India: Transmission dynamics of symptomatic vs. asymptomatic infections," medRxiv, Sep. 2020, doi: 10.1101/2020.09.17.20196501.

[41] S. L. Priyadarsini, M. Suresh, and D. Huisingh, "What can we learn from previous pandemics to reduce the frequency of emerging infectious diseases like COVID-19?," Glob. Transitions, vol. 2, 2020, doi: 10.1016/j.glt.2020.09.003. 